\title{
Bioethics in the Training and Management of Sport
}

\section{Iva Miteva ${ }^{a}$}

\begin{abstract}
The aim of this paper is to examine the opportunities and problems presented by the increasing availability of technologies which is capable of enhancing sporting performance and identifying the risks in sports for creating educational and sports management practices to lead with justice, honesty, and beneficence. A questionnaire is developed to ascertain the social determinants and risks of health aspects of sports in a sample of professionals and amateurs. Descriptive and correlation analysis were used for data analysis. The study identifies health risks connected with the intensive loading of locomotors system, poor working conditions which cause working trauma and diseases as well as low level of health culture regarding the effects of performance-enhancing drugs including anabolic steroids, stimulants, human growth hormone, and supplements. There are arguments that the quality of sport education will be achieved by addressing the study of sports ethics coupled with a study of management and law.
\end{abstract}

\section{Keywords}

Profession, professionalization, recognition of professional risks, sport management, ethics in sport

The policy of strengthening of quality physical education and sport needs an appraisal of approaches to manage the challenges in sports medicine. The literature review shows that the ethical issues in sport are very challenging all over the world. Information on the WHO (World Health Organization) Process for a "Global Strategy on Diet, Physical Activity and Health" indicates a clear advantage to the study of sports ethics coupled with a study of management and law. Discussions of the strategy at a lot of scholarly forums suggest that sport managers, coaches, and executives all need to have a solid grasp of the ethical decision-making process to perform their duties honestly, professionally, and ethically, and to deal with significant ethical and legal concepts widely used in sports (World Health Organization [WHO] 2004).

Sports morality and ethics are much debated topics latterly in Bulgaria. The intense debate focuses on the ethical dimension of the sport and how professionals produce a quality of experience that is evaluated as good in itself. Prevailing opinion is that there is a practical reason for studying sports ethics. A comprehensive analysis based on data of recent years found that professional sports organizations as a whole need to follow ethical guidelines and make ethical decisions. They face increasing competition in a rapidly changing environment and they must understand that ethical principles, rules, and standards have long-term benefits to individual, team, and organizational success (Kickbusch and Gleicher 2014).

\footnotetext{
aMedical University of Sofia, Bulgaria

Correspondent Author:

Iva Miteva, Sofia 1527, Bialo More Str. 8, University Hospital "Queen Joanna", IV floor

E-mail: mitevai@ymail.com
} 
Sports are so important that the different parties should collaborate to maintain the integrity of the sport and the greater glory, pride, and self-esteem of the team, city, and the country itself. Sports, just as any other endeavor, place much emphasis on rules and regulations. As indicated in the "Report of the International Bioethics Committee of UNESCO (United Nations Educational, Scientific, and Cultural Organization) (IBC): On Social Responsibility and Health" (United Nations Educational, Scientific, and Cultural Organization [UNESCO] 2010), the ethical standards serve as the basis for sports ethics. This topic is a fundamental introduction for encouraging an environment that highlights the essence of sports: fair play, character development, and excellence.

According to the "National Strategy for the Development of Physical Educational Sports in the Republic of Bulgaria 2010-2020" (Council of Ministers of Bulgaria 2009), ethics in sport requires four key virtues: fairness, integrity, responsibility, and respect. Starting position is the view that the sports professionals and amateurs need to follow established rules and guidelines, to gain an advantage by means of skills, to take responsibility for their actions, and show respect for teammates, opponents, coaches, and officials. Evidence from past research shows that the ethical approach to sport proceeds from the assumption that healthy competition in sport is a means of cultivating personal honor, virtue, and character.

\section{AIMS AND METHODS OF THE RESEARCH}

The aim of this study is to examine the opportunities and problems presented by the increasing availability of technologies capable of enhancing sporting performance and identifying the risks in sports for creating educational and sports management practices to lead with justice, honesty, and beneficence.

Data were collected from the 2012/2013 survey. Based on an extensive review of the literature, a questionnaire is developed to consider the social determinants and risks of health aspects of sports. The questionnaire is designed to explore issues relating to demographic data, diagnostic, preventive, rehabilitative, and performance enhancement services in sport. The tool requires the respondents to score items according to the frequency of occurrence of health risks and how they are handled. The sample for the study was composed of two respondent groups engaged in sport-100 professionals and 300 amateurs. The questionnaire was self-completed by all the participants in the study. The results of the inquiry are supported with an objective analysis. Descriptive and correlation analysis were used for data analysis.

\section{RESULTS AND DISCUSSIONS}

Sport is defined as all forms of physical activity which, through casual or organized participation, aim at expressing or improving physical fitness and mental wellbeing, forming social relationships or obtaining results in competition at all levels (Marmot and Allen 2014). To be active in sport requires those that are engaged to be up to date on the rules and regulations governing the sport and taking responsibility for their performance (Cahn and Markie 2008).

The research interests include the areas of gender, race, and ethnicity in sport, women in sport, multiculturalism and ethics in sport management. When analyzing the demographic data, there seem to be strong differences according to the characteristics of the respondent groups. A close connection with gender, age, and level of education was identified.

Gender differences were significant among professionals. Men exercise or play sport more than women. Men were $77.4 \%$, while women-less than a quarter of the professional group (22.6\%). The study found that the majority of the participants actively engaged in sports consist of men in the 15-25 and 25-35 age groups. The amount of sports activity decreases with age: 
(1) There are $64.3 \%$ of the professionals aged 15-25 years old;

(2) There are $20 \%$ of the professionals aged $25-35$ years old;

(3) There are $28.4 \%$ of the professionals aged over 35 years.

Those with high levels of education have increased levels of physical activity. The majority of respondents have secondary education and not a small part are with higher education:

(1) With secondary school education (62.1\%);

(2) With university education (28.4\%).

The comparative analysis of the frequency of accidents, based on data from official statistics and those identified from this survey proved the high number of unregistered and unannounced health disorders among professionals.

Among the issues addressed is the health risk connected with the intensive loading of locomotors system as well as poor working conditions which cause working trauma and diseases. Professionals with a very high physical activity are $71.3 \%$, those with a lot of every day stress are $50 \%$, and $68.2 \%$ of them notify of working trauma. A lot of cases with working trauma are associated with increased musculoskeletal distortion-26.4\%, and with fracture-21.8\%.

The study identifies negative professionally factors that involves a certain health risk:

(1) Unkempt apparatus- $59 \%$;

(2) Noise and vibration- $4.5 \%$.

Engaging in sport is one of the ways of being physically active and the sports movement has a great influence on the level of health-enhancing physical activity in the general population. The demographic data for the group of amateurs show: (1) $65.3 \%$ of them are men and $34.7 \%$ are women; (2) $39.1 \%$ of the respondents are under 25 years and $40.8 \%$ of them are between 25 and 35 years. Most of the amateurs are with high university education (59.3\%), and those with secondary school education are in the second place. By professional statute, most of them are lawyers and students.

As to the sports activity, the study identifies that for $76.4 \%$ of the amateurs, this activity is essential for their health and wellbeing. A quarter of them by means of sports activity intend to make a fine figure and their behavior is associated with obesity and lose overweight. As to the duration of the activity, the results are similar to those in the group of professionals:

(1) There are $62.7 \%$ amateurs active more than five years;

(2) There are $22.4 \%$ amateurs active from two to five years;

(3) There are $14.6 \%$ amateurs active for one year now.

Amateurs were asked to report the number of days over the week that they were physically active. The intensity of sports activity among the amateurs shows that $41.8 \%$ of them are active every day and $33.3 \%$ of them are up to three times per week. Leading cause of this intensity of sports activity is the experience of real and worrying problems in relation to issues such as overweight and obesity, self-esteem and life satisfaction. Forty-two point two percent of the amateurs with high university education intend to make a fine figure while those with lower level of education are only $24 \%(\mathrm{p}=.034)$.

The study was intended to discuss how the health culture makes an impact on the behavior of respondents. Results suggest a low level of health culture in terms of the effects of taking performance - enhancing drugs including anabolic steroids, stimulants, human growth hormone, and supplements among both professionals and amateurs. It was found a low level of health culture among both groups - only $7.1 \%$ of them are well-grounded in the enhancing drugs from a reliable resource-the scientific literature.

The study shows that enhancing drugs are also of frequent occurrence among those that are engaged professionally with sports $-46.8 \%$ are taking drugs 
more than five years and $25.5 \%$ are under five years. Fifty-two percent from the group of professionals are not acquainted with the risks of performance-enhancing drugs and the key factors that influence people's health and well-being. As was the case with professionals, most of the amateurs are also taking enhancing drugs- $-41.9 \%$ of them.

Because of an insufficient appreciation of the specific risks among the active professionals, the author intends to contribute in taking on this challenge to initiate a teaching module in the undergraduate program for students in different medical specialties. The initiative is in line with the policy of the WHO Regional Office for Europe (2011): "Promoting Sport and Enhancing Health in European Union Countries: A Policy Content Analysis to Support Action". The aim is to teach students about the pragmatic, medical, and social complexity of sports and how to address the systematic challenges by building greater awareness and understanding of the critical moral, ethical, and social dimensions of sports medicine (WHO Regional Office for Europe 2011; Trudeau and Shephard 2008).

Sports ethics is concerned with what is the right thing to do in sports. Any individual faced with an ethical or moral decision must make that decision based on certain guiding principles. Modules on sports medicine will provide strong evidence and data that will support students with the essential skills, a professional in sports needs to make ethical decisions, and the necessary tools to develop ethical models in the sports management field that the sports management professionals can use as a platform to make ethical decisions.

\section{CONCLUSIONS}

There are arguments that sports medicine bridges the gap between science and practice in the development of principle-centered leadership practices, and can serve as a good introduction to the many ethical dilemmas frequently arising within the context of sport management. Strengthening the quality of sport education will be achieved by addressing the study of sports ethics coupled with a study of management and law. There is a need for more well-trained sports professionals. In order to fully realize the benefits of sports ethics in the prevention, diagnosis, and treatment of disease, sports medicine must engage in an effective and principled partnership with bioethics (Cecchini et al. 2010; Hartmann 2010).

As the Bulgarian health care system undergoes fundamental and rapid reforms, medical curricula in health care policy must keep pace. The Faculty of Public Health is dedicated to improving the role bioethics plays in sport by building greater awareness and understanding of the critical moral, ethical, and social dimensions of sports medicine, in compliance with the requirements in the Final Report of the Commission on Social Determinants of Health: "Health Equity through Action on the Social Determinant of Health" (Commission on Social Determinants of Health 2008). By addressing neglected incentives, the results of this study led to new initiatives with a focus on improving the ethical literacy of the population, especially young people, how best to inspire and increase moral understanding, analytical thinking in the moral domain, and professional integrity of sport.

\section{References}

Cahn, St. M. and P. Markie. 2008. Ethics: History, Theory, and Contemporary Issues. 4th ed. New York: Oxford University Press.

Cecchini, M., F. Sassi, J. A. Lauer, Y. Y. Lee, V. Guajardo-Barron, and D. Chisholm. 2010. "Tackling of Unhealthy Diets, Physical Inactivity, and Obesity: Health Effects and Cost-effectiveness." Lancet 376(9754):1775-1784.

Commission on Social Determinants of Health. 2008. CSDH Final Report: Closing the Gap in a Generation: Health Equity Through Action on the Social Determinant of Health. WHO, Geneva, Switzerland.

Council of Ministers of Bulgaria. 2009. National Strategy for the Development of Physical Educational Sports in the Republic of Bulgaria 2010-2020. Council of Ministers of 
Bulgaria.

Hartmann, H. 2010. "European Organizations Connecting Sport, Health and Culture From Citizens Point of View." Presented at the Seminar of the International Sport and Culture Association: "Sport and Culture in Dialogue". October 1-3, Local Citizens Panel, Zagreb, Croatia.

Kickbusch, I. and D. Gleicher, eds. 2014. Smart Governance for Health and Well-being: The Evidence. WHO Regional Office for Europe.

Marmot, M. and J. Allen. 2014. "Social Determinants of Health Equity." American Journal of Public Health 104(S4):S517-S519.

Trudeau, F. and R. J. Shephard. 2008. "Physical Education, School Physical Activity, School Sports and Academic Performance." International Journal of Behavioral Nutrition and Physical Activity 5:10.

UNESCO (United Nations Educational, Scientific, and Cultural Organization). 2010. Report of the International Bioethics Committee of UNESCO (IBC): On Social Responsibility and Health. Social and Human Sciences Sector, Division of Ethics of Science and Technology, Bioethics Section, UNESCO.

WHO (World Health Organization). 2004. Global Strategy on Diet, Physical Activity and Health. WHO, Geneva. Retrieved October 30, 2013 (http://www.who.int/diet physicalactivity/en/).

WHO Regional Office for Europe. 2011. Promoting Sport and Enhancing Health in European Union Countries: A Policy Content Analysis to Support Action. WHO Regional Office for Europe.

\section{Bio}

Iva Miteva, Ph.D., assistant professor, Department of Occupational Health, Faculty of Public Health, Medical University of Sofia; research fields: sports medicine, risk management, and bioethics. 